\title{
Detecting nonlinearity in time series driven by non-Gaussian noise: the case of river flows
}

\author{
F. Laio ${ }^{1}$, A. Porporato ${ }^{2}$, L. Ridolfi ${ }^{1}$, and S. Tamea ${ }^{1}$ \\ ${ }^{1}$ Dip. Idraulica, Trasporti ed Infrastrutture Civili, Politecnico di Torino, Torino, Italy \\ ${ }^{2}$ Dept. of Civil and Environmental Engineering, Duke University, Durham, NC, USA
}

Received: 14 June 2004 - Revised: 29 September 2004 - Accepted: 4 October 2004 - Published: 26 October 2004

Part of Special Issue "Nonlinear deterministic dynamics in hydrologic systems: present activities and future challenges"

\begin{abstract}
Several methods exist for the detection of nonlinearity in univariate time series. In the present work we consider riverflow time series to infer the dynamical characteristics of the rainfall-runoff transformation. It is shown that the non-Gaussian nature of the driving force (rainfall) can distort the results of such methods, in particular when surrogate data techniques are used. Deterministic versus stochastic (DVS) plots, conditionally applied to the decay phases of the time series, are instead proved to be a suitable tool to detect nonlinearity in processes driven by non-Gaussian (Poissonian) noise. An application to daily discharges from three Italian rivers provides important clues to the presence of nonlinearity in the rainfall-runoff transformation.
\end{abstract}

\section{Introduction}

The processes that control the rainfall-runoff transformation contain nonlinear deterministic components: examples of such processes include the unsteady flow in the channel network, the infiltration in the vadose zone, the control of vegetation on transpiration, and the presence of several threshold processes governing the dynamics such as interception, deep infiltration, etc. (Porporato and Ridolfi, 2003). However, the presence of nonlinear components in a system does not imply that all signals measured from the system are themselves nonlinear (e.g. Schreiber and Schmitz, 2000). For example, the local nonlinearities can act in opposite directions and compensate one another, or they can be quantitatively small compared to other stochastic components.

As a result, despite a remarkable amount of literature on this topic (see Porporato and Ridolfi (2003) for a review), the linearity or nonlinearity of the rainfall-runoff transformation remains an open question. For example, some researchers reported a higher degree of nonlinearity in small catchments, where overland flow and unsaturated subsurface

Correspondence to: F. Laio

(francesco.laio@polito.it) flow are predominant (Pilgrim, 1976; Wang et al., 1981), while other studies supported opposite conclusions (Robinson et al., 1995; Goodrich et al., 1997). Other works linked the evidence of nonlinearity to the value of discharge, detecting a stronger nonlinearity during periods of high (Liu and Brutsaert, 1978) or low (Pilgrim, 1976) discharges. Still other authors related the nonlinearity to the time scale under analysis, observing that linearity dominates in the annual and monthly discharges (Rao and Yu, 1990; Chen and Rao, 2003). It can be certainly stated that the problem deserves further investigations, also because of its practical implications in the formulation of efficient models for forecasting and simulating the evolution of river flows (Amorocho, 1967; Weiss, 1977; Rogers, 1982; Pilgrim and Cordery, 1993; Murrone et al., 1997; Chen and Rao, 2003).

Nonlinearity in the rainfall-runoff transformation is investigated here in a univariate setting, by considering daily river flow time sequences. To this aim, we benefit from phase space reconstruction methods, originated in the framework of nonlinear time series analysis (Takens, 1981; Sauer et al., 1991; Abarbanel, 1996; Kantz and Schreiber, 1997; Schreiber, 1999), which provide the possibility to recover the dynamics from univariate time series. Several applications of these techniques in the hydrological field have been proposed (see the review papers by Sivakumar (2000) and Sivakumar (2004)). Clues to the existence of nonlinear deterministic components have emerged, but great caution is necessary to distinguish real nonlinearity from spurious results (Schreiber, 1999). In fact, each method to detect nonlinearity in a time series has its peculiarities and drawbacks especially when applied to real data (Porporato and Ridolfi, 1997).

The non-Gaussianity of the data and the presence of a seasonal component are possible causes of distortion producing misleading results of the nonlinearity tests. The scope of this paper is to draw the attention to the effects of these factors, with particular regard to non-Gaussianity, and to caution against the widespread use of surrogate data techniques, which are prone to produce spurious results in the presence 

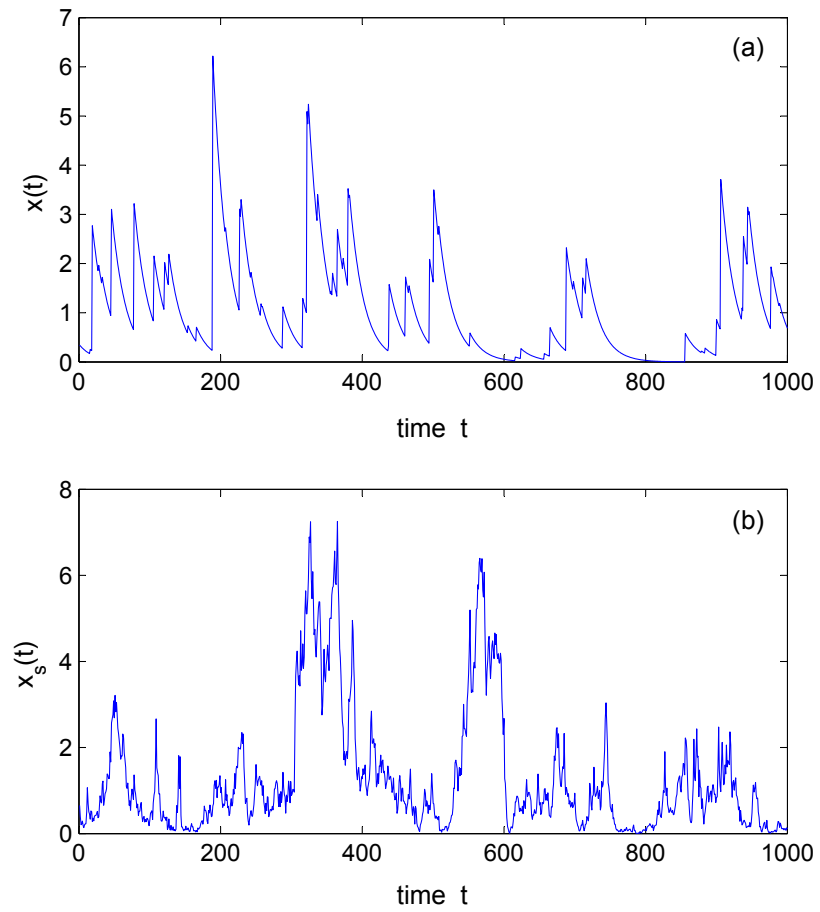

Fig. 1. Samples from (a) a linear shot noise model (1) and (b) a correspondent surrogate series.

of these effects (Sect. 2). Deterministic versus Stochastic (DVS) plots (Casdagli, 1991), conditionally applied to the phases of decay of the time series, are shown to be a versatile and robust tool to discriminate between linear and non-linear time series, even when the Poissonian (or, in general, jump) components dominate, as often happens in daily discharge time series (Sect. 3). An application to three daily discharge time series from Italian rivers confirms these findings, and demonstrates the practical usefulness of the tool (Sect. 4).

\section{Nonlinearity and non-Gaussianity}

Some confusion between nonlinearity and non-Gaussianity arises from the widespread adoption of surrogate data techniques in testing for nonlinearity. Surrogate data are time series generated by a stationary Gaussian linear stochastic process (i.e. an autoregressive moving-average, or ARMA, process) and possibly distorted through an invertible, static, nonlinear observation function in such a way that they have similar spectrum (or autocorrelation function) and probability density function to the original time series to test (Theiler et al., 1993; Schreiber and Schmitz, 2000; Kugiumtzis, 2002). They are used according to the following logical steps: (i) a significant set of surrogate series is artificially generated, for example using the TISEAN 2.1 package (Hegger et al., 1999); (ii) statistics sensitive to nonlinearity are determined for both surrogate and original series; and (iii) if the statistics of the surrogate are significantly different from the original series, the null hypothesis that the orig- inal data are generated by an ARMA process is rejected. The tests differentiate according to the nonlinear statistics used in the second step. This is a key point (Schreiber and Schmitz, 1997, 2000) and several criteria have been proposed; e.g. predictability (Chang et al., 1995; Basu and Foufoula-Georgiou, 2002), characteristics of the exceptional events (Kaplan, 1994; Chang et al., 1995; Barnett et al., 1997), structure of the volatility (Ashkenazy et al., 2003), and time reversibility (Schreiber and Schmitz, 1997).

In the last years the surrogate data approach has become so popular that the surrogate series have often become synonymous with linearity. In reality, this is correct only if the random component of the dynamics is known to be a Gaussian noise, which is seldom the case for daily discharge time series. Other types of noise can produce misleading results: an example is provided by a linear shot noise process (e.g. Cox and Isham, 1986; Laio et al., 2001)

$\frac{\mathrm{d} x(t)}{\mathrm{d} t}=-x(t)+N_{p}(t)$,

where $N_{p}(t)=\sum_{-\infty}^{t} \delta\left(t-\tau_{i}\right) \Delta_{i}$ is white Poisson noise, i.e. a marked point process where both the events' inter-arrival times, $\tau_{i}-\tau_{i-1}$, and the jump sizes, $\Delta_{i}$, are exponentially distributed $(\delta(\cdot)$ is the Dirac delta function). The process is linear, but the series is evidently very different from its surrogate counterpart (see Figs. 1a and b), since no ARMA process, even nonlinearly distorted with a static transformation, can reproduce the jump-decay structure of the original process.

These differences can easily lead to a rejection of the test null hypothesis, even if the process is linear: in fact, when surrogate data techniques are adopted, the null hypothesis is Gaussian-linearity rather than linearity alone. For example, the time series in Fig. 1a exhibits a clear temporal irreversibility, that contrasts with the reversibility characteristic of linear Gaussian processes (Weiss, 1975; Diks et al., 1995; Daw et al., 2000). In fact, the simple third order statistic $r$ proposed by Schreiber and Schmitz (2000) to test for reversibility produces a value of $r=0.34$ for the linear shot noise process, well above the maximum value of $r=0.095$ obtained from 20 surrogate series. The test works correctly, since it allows one to reject the null hypothesis of linearGaussianity, but the results should not be misinterpreted as a clue to nonlinearity. As a consequence, in all cases when the presence of non-Gaussian noise is suspected, the surrogate data technique (as presently formulated) is useless to detect possible nonlinearities. Similar considerations apply to other highly regarded tests for nonlinearity, like the BDS test (Brock et al., 1996), which are based on fitting a linear Gaussian model to the data and analyzing the residuals (see also Barnett et al., 1997).

Going back to the river flow time series, the basic point is then to understand how important the non-Gaussian component of the noise is: a visual inspection of the time series, and a revision of the literature on synthetic streamflow generation (e.g. Lawrance and Kottegoda, 1977), can lead to the consideration that the degree of Gaussianity is linked to the 

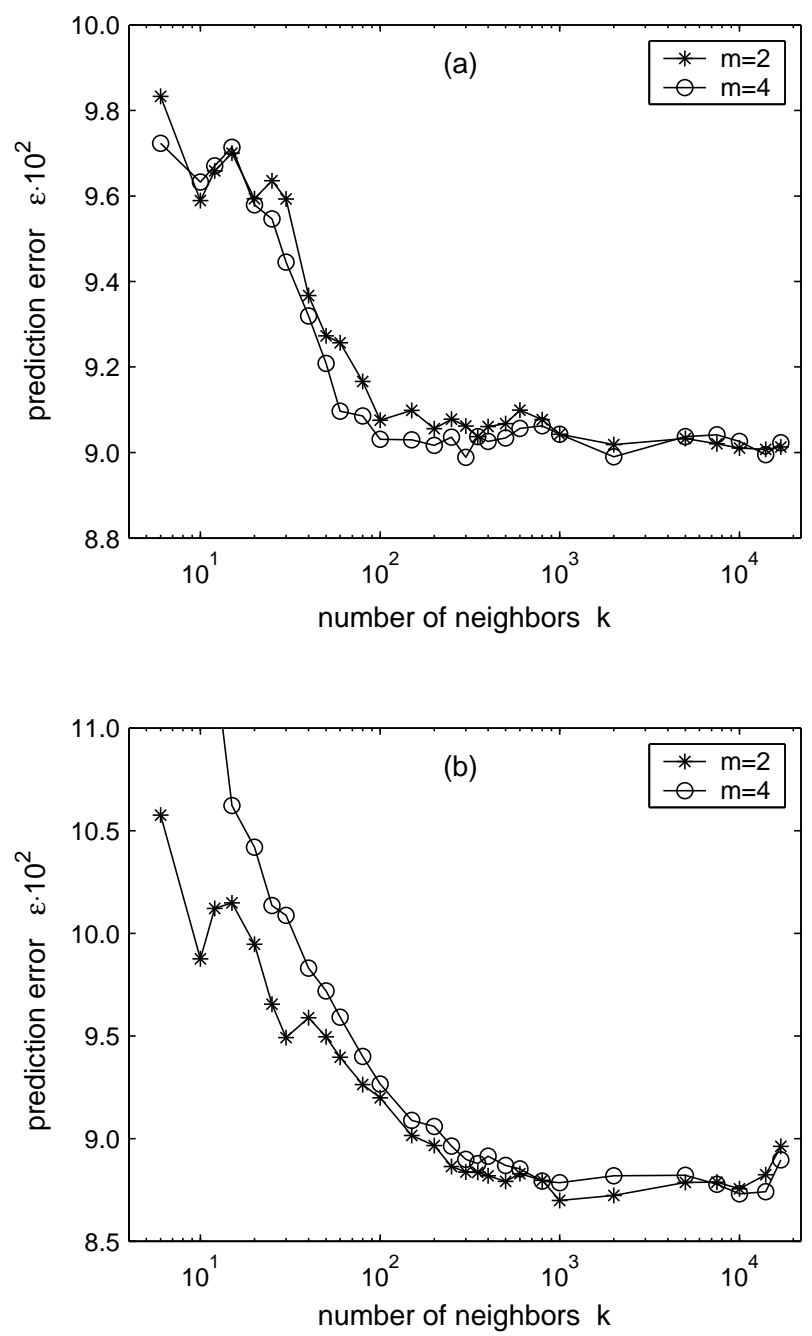

Fig. 2. DVS plots for (a) a linear and (b) a nonlinear shot noise process. The size of the testing set is 1000 data, $\tau=1$ and $T=1$.

relation between the aggregation time of the data, $T_{a}$, and the concentration time of the catchment, $T_{c}$. When $T_{a} \gg T_{c}$ (e.g. monthly discharges) the time series is smooth and does not have evident characteristics that contradict an eventual linear Gaussian dynamics; in fact, ARMA process are often employed to generate monthly streamflow data (Salas, 1993). In contrast, when $T_{a} \simeq T_{c}$ (e.g. daily discharges for mediumsize basins) the time series has a structure that resembles a stochastic jump process: the intermittent occurrence of rainfall produces jumps that are followed by recession curves. The series is irreversible (Lawrance and Kottegoda, 1977), and shot noise models are often used to generate the data (Weiss, 1977; Murrone et al., 1997). Finally, when $T_{a} \ll T_{c}$ (e.g. hourly discharges) the discharge increments cannot be seen as instantaneous jumps, but the series is still dominated by the random and intermittent occurrence of rainfall events, which maintain a high degree of irreversibility in the time series. On these grounds, it is clear that the nonlinearity tests using surrogate time series are useful only when $T_{a} \gg T_{c}$, while they can lead to erroneous conclusions in the other two cases (e.g. Livina et al., 2003).
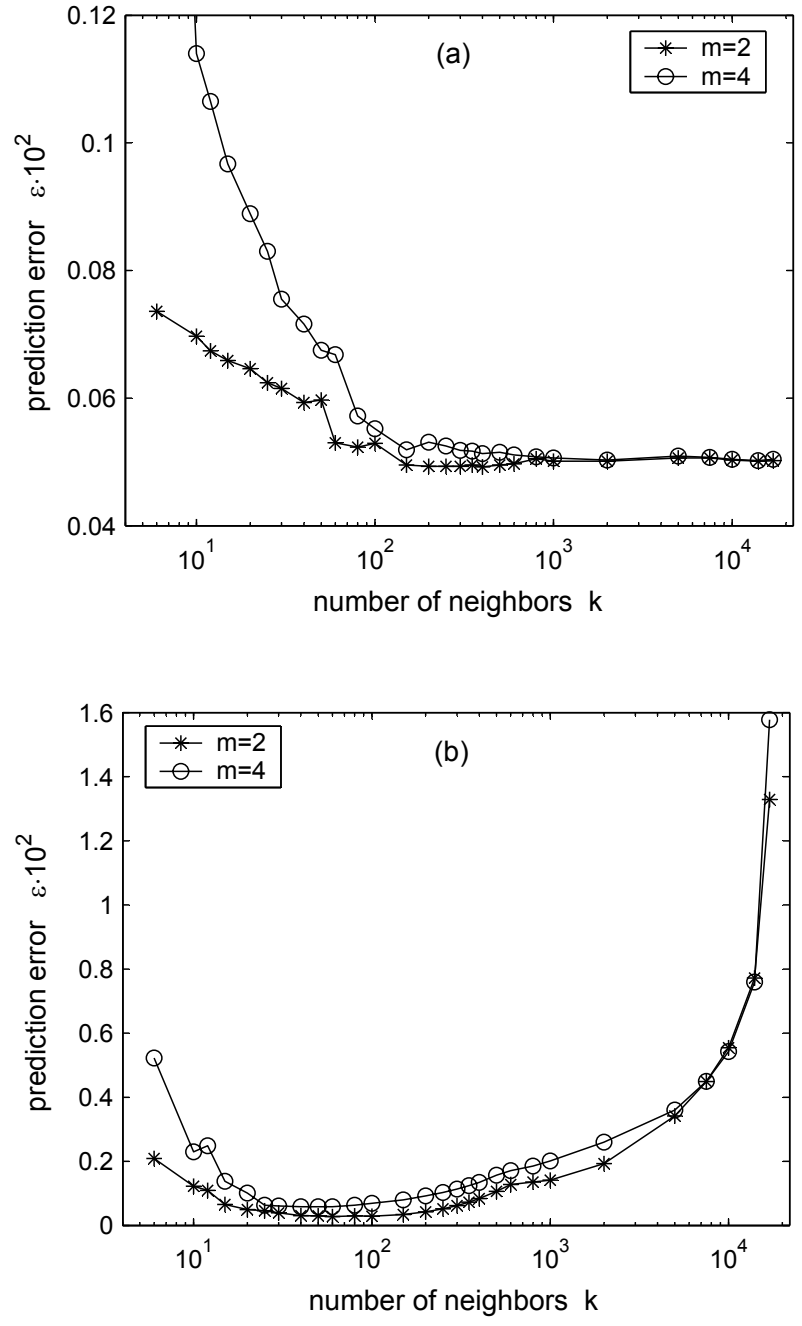

Fig. 3. recDVS plots for the same (a) linear and (b) nonlinear shot noise process as in Fig. 2.

\section{Detecting nonlinearity by DVS plots}

Deterministic versus stochastic (DVS) plots have been proposed by Casdagli (1991) to explore time series dynamical properties and to detect the presence of chaos in the underlying system. In this work they are employed as an investigating tool which allows to avoid some problems affecting other nonlinearity tests. The basic idea behind the use of DVS plots to test for nonlinearity is to compare linear and nonlinear models to see which more accurately predicts the future. Suitable linear and nonlinear prediction models need to be defined. Following Casdagli (1991), the dynamics is first reconstructed in the phase space from the measured time series $\left\{x_{i}\right\}, i=1, . . N$, by using Takens' delay time method, with properly chosen embedding dimension $m$ and delay time $\tau$. Local linear models,

$x_{i+T}=a_{0}+a_{1} x_{i}+a_{2} x_{i-\tau}+\ldots+a_{m} x_{i-(m-1) \tau}$,

where $T$ is the forecast time, are then fitted using the $k$ nearest neighbors of the regressor vector $\left[x_{i}, . ., x_{i-(m-1) \tau}\right]$. 

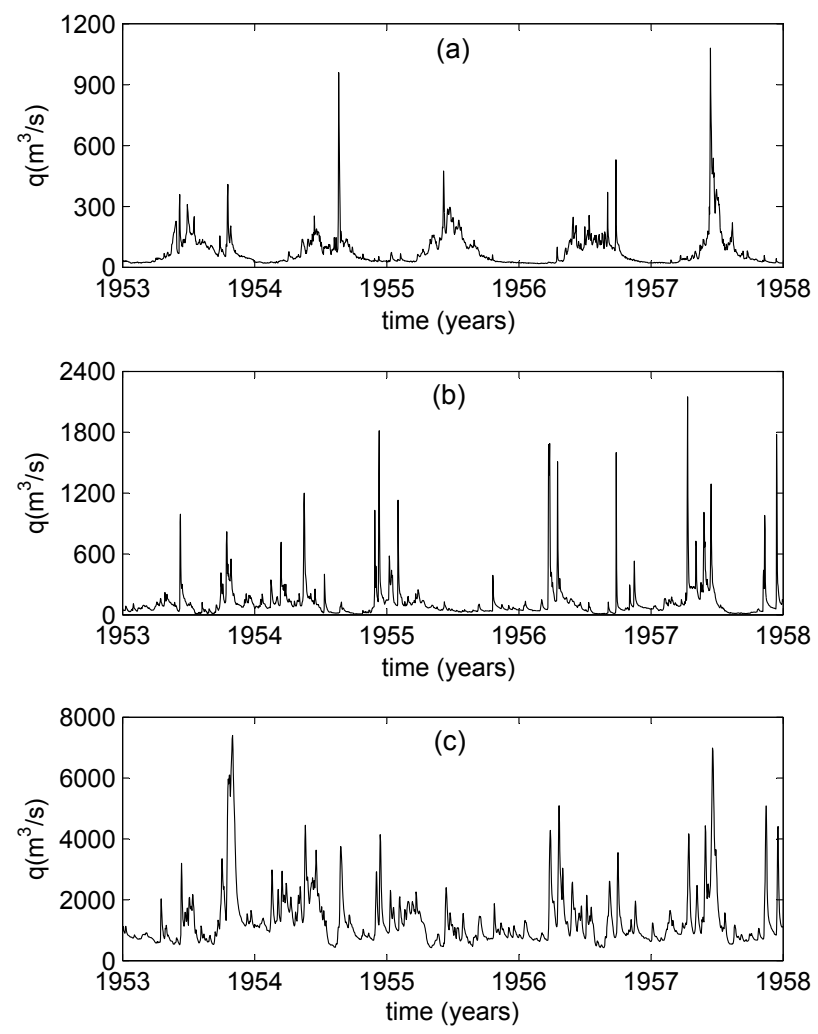

Fig. 4. Samples of daily discharge time series for (a) DB, (b) TA and (c) PO rivers.

When $k$ is low, the forecasting model is the first order nonlinear prediction method of Farmer and Sidorowich (1987), while for $k$ equal to the total size of the fitting set one finds a globally linear model. A measure of the prediction error $\epsilon$ can be calculated on a testing set for varying $k$, and plotted against $\log (k)$ to obtain the DVS plot: a decreasing plot indicates a better aptitude of global linear models to predict the dynamics, and it is a clue to an underlying linear stochastic system; a curve monotonically increasing or with a minimum at a low value of $k$ shows evidence of low dimensional chaos; finally, the presence of a minimum for intermediate $k$ values suggests a nonlinear stochastic behavior of the dynamical system. Following the suggestion of Casdagli (1993), the mean absolute error rather then the mean squared error is taken as an indicator of the prediction accuracy, basically for its lower sensitivity to outliers.

The DVS technique cannot be considered a rigorous statistical test, since no test statistics is produced which allows a univocal acceptance or rejection of the null hypothesis of linearity. However, the DVS method is more general than other techniques that are used in the literature, and it is less prone to produce erroneous results, except for particular cases (Theiler et al., 1993). For example, tests which compare linear and nonlinear prediction in an apparently formal manner (e.g. Chang et al., 1995; Schreiber and Schmitz, 1997; Kugiumtzis, 2002), have the drawback of requiring to fix a priori the number of neighbors $k$, with different choices possibly leading to opposite results in terms of nonlinearity detection. The robust and qualitative DVS technique seems more appropriate, at least when the object of the nonlinearity analysis are short and noisy real data time series, which are more liable to produce ambiguous results.

As a check of the ability of the DVS technique to detect nonlinearity when non-Gaussian noise is present, we apply the DVS method to the linear shot noise model (1) (see also Fig. 1). DVS plots take the form of Fig. 2a: the plot is monotonically decreasing, proving that the best predictions are obtained with a global linear model. DVS plots are thus able to recognize the linear nature of the underlying dynamical system, without being biased by the presence of non-Gaussian noise.

As a counterproof, the DVS method is also applied to another artificial time series, similar to the linear shot noise process (1) but characterized by a nonlinear loss function,

$\frac{\mathrm{d} x(t)}{\mathrm{d} t}=-x^{5}(t)+N_{p}(t)$.

The best predictions are no more found at the right extreme of the DVS plots (Fig. 2b) but the minimum of the prediction error is not as pronounced as it would be expected for a markedly nonlinear process. The problem is that the process is dominated by the stochastic component (the random jumps), while the nonlinearities are somewhat hidden in the recessions. In fact, the processes generated by (1) and (3) do not show striking differences at a visual inspection. However, these nonlinearities can be very relevant from a physical viewpoint: for example, they would be an evident clue to the nonlinearity of the rainfall-runoff transformation if they were detected in discharge time series. The relevance of the topic demands special tools for the detection of nonlinearity in time series driven by non-Gaussian noise. One of these tools is found in a conditional form of the DVS technique, as detailed below.

The basic idea behind the method is to look for nonlinearities where they are expected to be, i.e. in the recession curves. Linear and nonlinear predictions are thus carried out by choosing the $k$ nearest neighbors such that they all belong to recessions, which are loosely defined here by imposing the condition $x_{i}>x_{i+T}$. The prediction error $\epsilon$ is then evaluated on the set of points of the testing set belonging to recession curves. By measuring $\epsilon$ for varying $k$ one gets a DVS plot which is targeted on the recession curves (in short, a recDVS plot). Application of the recDVS technique to the linear shot noise process (1) is reported in Fig. 3a and shows a well behaved decreasing curve from small to large $k$ 's, without substantial differences from the classical DVS plot. An analogous recDVS plot for the nonlinear shot noise process (3) produces the results in Fig. 3b: the mean absolute error has a clear minimum for $k$ below 100 , providing a relevant clue to nonlinearity of the process, that was not so evident in the classical DVS plot. The method is therefore unbiased and it is more powerful than the classical DVS technique when applied to time series driven by Poissonian 

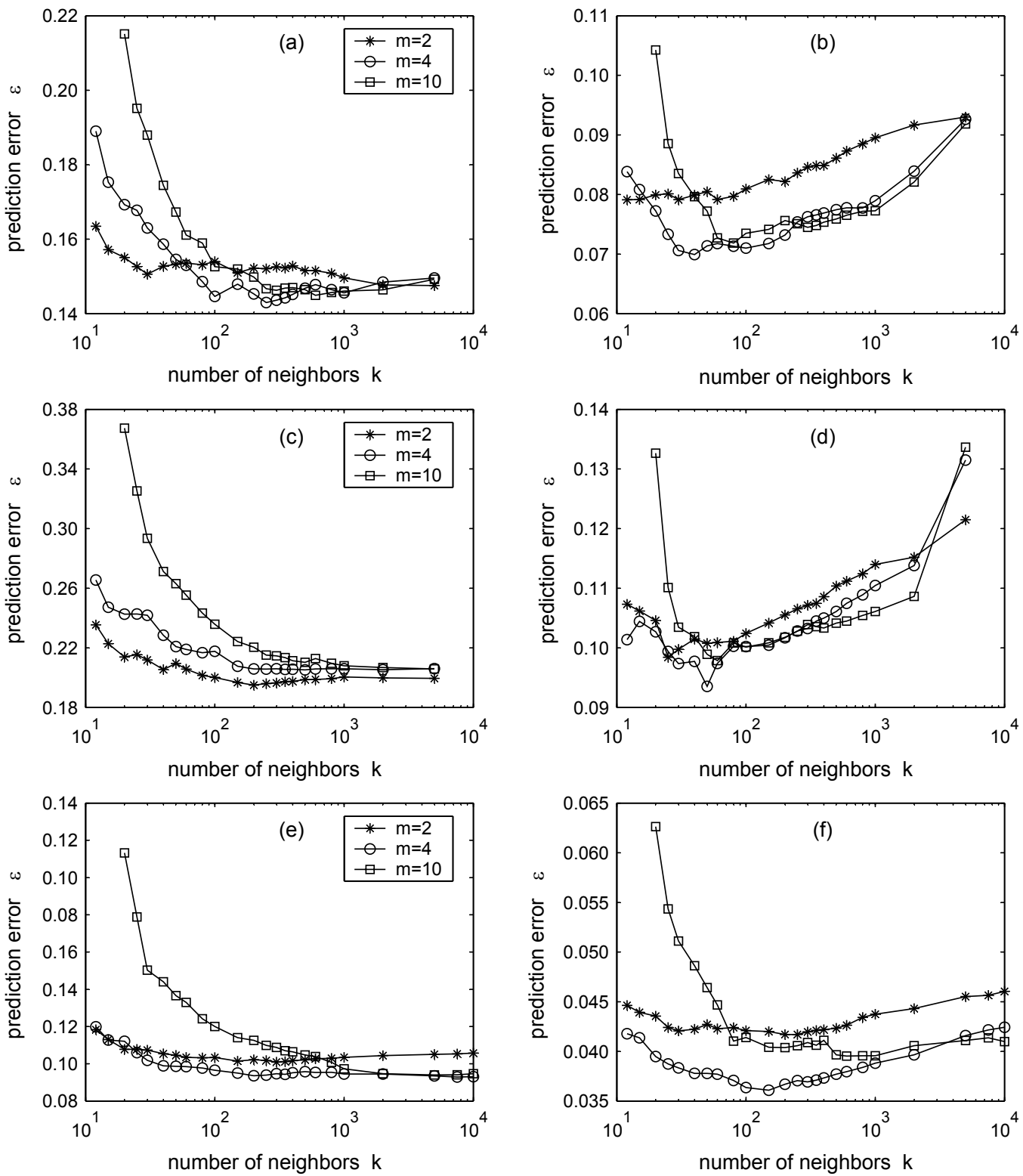

Fig. 5. DVS (left panels) and recDVS (right panels) plots for standardized daily discharges of (a) and (b) DB, (c) and (d) TA and (e) and (f) PO rivers. For all cases, the size of the testing set is 2 years, $\tau=1$ day and $T=1$ day.

noise, while it provides analogous results to the DVS diagram when the noise is Gaussian. The $\epsilon$ values in Fig. 3 are consistently lower than those in Fig. 2 for two reasons: (i) the random jumps in the trajectory are not considered in the forecast when applying the recDVS technique, and (ii) the predictions in Fig. 3 take advantage of the prior knowledge that $x_{i+T}$ will be lower than $x_{i}$, an information which is obviously unavailable in real forecasting.

\section{Application to river flows}

Three daily river flow time series are considered (see Fig. 4), pertaining to the Dora Baltea river at Tavagnasco (DB), the
Tanaro river at Montecastello (TA) and the Po river at Pontelagoscuro (PO). The same three rivers were selected as the object of investigation by Porporato and Ridolfi (2003), to which the reader is referred for details regarding the main features of the drainage basins and of the discharge time series. We remark here that the basin areas are $\sim 3300 \mathrm{Km}^{2}$ (DB), $\sim 8000 \mathrm{Km}^{2}$ (TA) and $\sim 70000 \mathrm{Km}^{2}$ (PO); for the DB and TA rivers the concentration time can be estimated to be close to the aggregation time of the time series (1 day), $T_{a} \simeq T_{c}$, while for the PO river one has $T_{a}<T_{c}$. As expected from the classification in Sect. 2, the jump-recession structure of the time series is predominant for the $\mathrm{DB}$ and TA rivers, while for the $\mathrm{PO}$ river the sudden jumps in the trajectories are replaced by slower rises of discharge in 

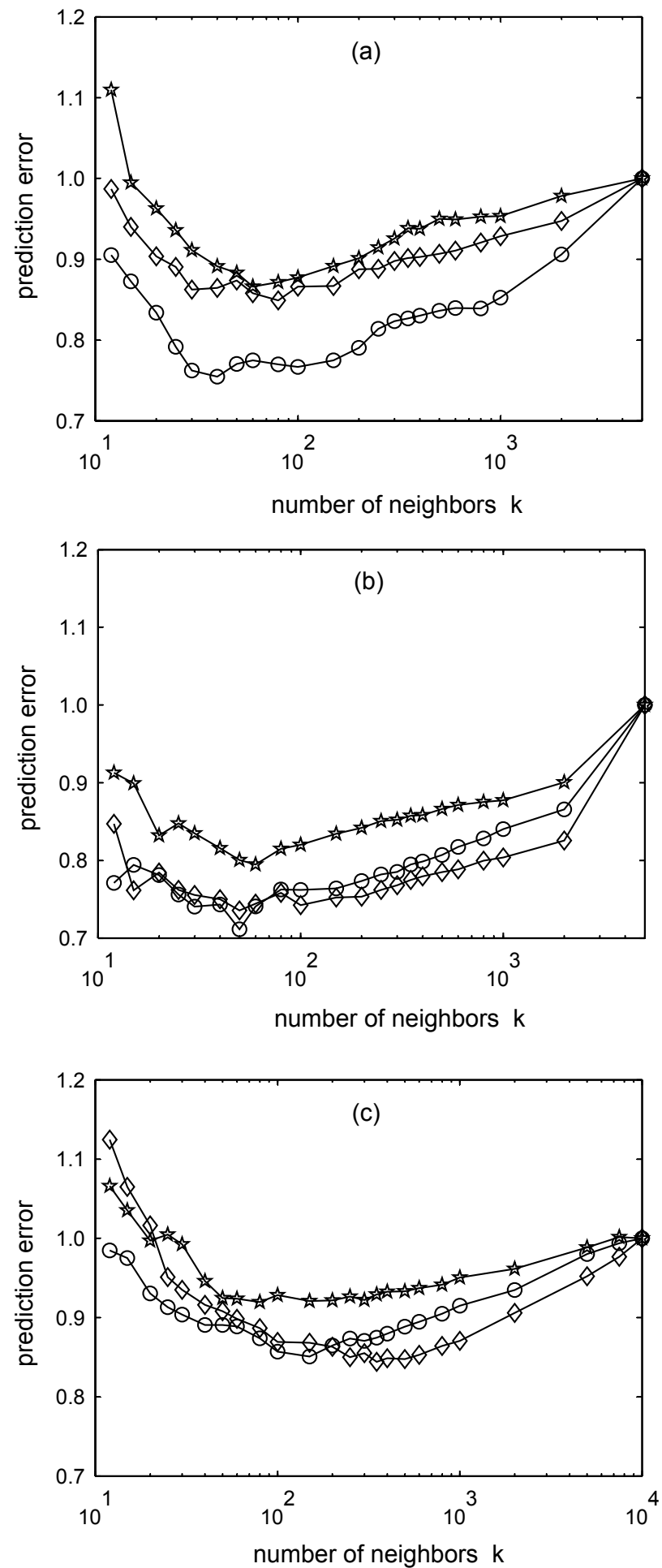

Fig. 6. recDVS plots for daily discharges of (a) DB, (b) TA and (c) PO rivers. In all cases the size of the testing set is 2 years, $m=4$, $\tau=1$ and $T=1$ day. Open circles correspond to the original curves in Fig. 5, open diamonds to time series deseasonalized in the mean, and stars to seasonally standardized data. In order to facilitate the comparison, all the curves are reported to have $\epsilon=1$ at the right extreme. correspondence to the rain events; even in this case, the role of non-Gaussian noise is however relevant: in fact, recession curves are easily recognizable from the rising limbs of the hydrographs, demonstrating a lack of time-reversibility.

DVS and recDVS techniques are applied to the standardized discharge time series, with the results reported in Fig. 5 for typical values of the embedding dimension and delay time ( $m=2,4$ and 10 and $\tau=1$ day, see also Porporato and Ridolfi (2003)). When the classical DVS plots are employed (left panels in Fig. 5), one obtains rather ambiguous results, with a general tendency towards having a minimum of the prediction error for large values of $k$, or, in particular for the DB river, a gentle minimum for $k$ lower than 1000 . The results are rather robust with respect to changing $m, \tau$ or the prediction jump $T$, and agree fairly well with the findings of Porporato and Ridolfi (2003). When in contrast the recDVS technique is adopted (right panels in Fig. 5), one obtains much clearer results, with well-shaped minima for $k$ around 100, providing good evidence of nonlinear dynamics in the recession curves. Only for the PO river the results are slightly less definite, possibly due to role of the larger concentration time. It is noteworthy that daily river flows behave in a very similar manner to the nonlinear shot noise model (3), with an almost flat DVS plot and a clear minimum in the recDVS plot. This finding has important implications for the problem of selecting a suitable model for synthetic streamflow generation.

Before assuming as ascertained the presence of nonlinearity, a further point needs to be considered: the presence of a seasonal component in the dynamics can dilate the coherence times and mislead the nonlinearity tests, which are prone to mistake seasonality for nonlinearity (Theiler et al., 1993). Two ways for investigating if DVS plots are influenced by seasonality can be considered: an artificial linear time series with a superimposed seasonal component can be generated and tested for nonlinearity by using DVS techniques (Theiler et al., 1993); or one can remove seasonality from a real time series, and re-apply the DVS method to check if relevant differences appear between the original and deseasonalized time series. Both methods confirm that seasonality may produce a fictitious impression of nonlinearity, even for linear time series. However, the effect is not very strong: the superimposition of a sine wave to the linear model (1) causes an increase of the prediction error for large $k$ values in the recDVS plot, but the deviations of the DVS plots are not significant. Accordingly, Fig. 6 demonstrates that, even when seasonality is removed, a clear minimum for $k \sim 100$ is retained in the recDVS plots. Note that this result is robust with respect to the method used to remove seasonality: in fact, the open diamonds in Fig. 6 correspond to a removal of seasonality in the (monthly) mean, while the stars correspond to a seasonal standardization, i.e. to the removal of seasonality in the mean and variance, obtained by subtracting the monthly mean and dividing by the monthly standard deviation. The full deseasonalization has a stronger effect on the DVS plots, but the nonlinear signature of the dynamics remains evident. 


\section{Conclusions}

The main results of this paper, related to the detection of nonlinearity in non-Gaussian time series, are listed below. The topic is crucial for river flow time series, that at the daily time scale are typically characterized by a non-Gaussian behavior.

(i) Surrogate data techniques are inadequate to testing nonGaussian time series for nonlinearity: in fact, linear non-Gaussian systems are easily mistaken for nonlinear Gaussian dynamical systems.

(ii) DVS plots are more robust towards non-Gaussianity, but they show the opposite problem: nonlinear nonGaussian systems show a similar response as linear Gaussian systems, since the possible nonlinearities tend to be dominated by the stochastic component.

(iii) Nonlinearity is better detected by concentrating on those parts of the time series that are not disturbed by the jumps, namely the recession curves. A conditional DVS technique is then proposed, which is shown to produce very good results when applied to synthetic nonGaussian (Poissonian) time series.

(iv) At the time scale of the concentration time, the dynamics of river discharges show clear signatures of nonlinearity in the recession curves. Seasonality does not substantially modify this finding.

Edited by: B. Sivakumar

Reviewed by: H.-S. Kim and another referee

\section{References}

Abarbanel, H. D. I.: Analysis of observed chaotic data, Springer, Berlin, Germany, 1996.

Amorocho, J.: The nonlinear prediction problem in the study of the runoff cycle, Water Resour. Res., 3, 861-880, 1967.

Ashkenazy, Y., Havlin, S., Ivanov, P. C., Peng, C.-K., SchulteFrohlinde, V., and Stanley, H. E.: Magnitude and sign scaling in power-law correlated time series, Physica A, 323, 19-41, 2003.

Barnett, W. A., Gallant, A. R., Hinich, M. J., Jungeilges, J. A., Kaplan, D. T., and Jensen, M. J.: A single-blind controlled competition among tests for nonlinearity and chaos, J. Econometrics, 82, 157-192, 1997.

Basu, S. and Foufoula-Georgiou, E.: Detection of nonlinearity and chaoticity in time series using the transportation distance function, Phys. Lett. A, 301, 413-424, 2002.

Brock, W. A., Dechert, W. D., Scheinkman, J. A., and LeBaron, B.: A test for independence based on the correlation dimension, Econ. Rev., 15, 197-235, 1996.

Casdagli, M.: Chaos and deterministic versus stochastic nonlinear modeling, J. Roy. Stat. Soc. B, 54, 303-324, 1991.

Casdagli, M.: Exploring the continuum between deterministic and stochastic modeling, in: Time series prediction: forecasting the future and understanding the past, edited by: Weigend, A. and Gershenfeld, N., 347-365, Addison-Wesley, Reading, MA, 1993.

Chang, T., Sauer, T., and Schiff, S.: Tests of nonlinearity in short stationarity time series, Chaos, 5, 118-126, 1995.
Chen, H.-L. and Rao, A. R.: Linearity analysis on stationary segments of hydrologic time series, J. Hydrol., 277, 89-99, 2003.

Cox, D. and Isham, V.: The virtual waiting-time and related processes, Adv. Appl. Prob., 18, 558-573, 1986.

Daw, C. S., Finney, C. E. A., and Kennel, M. B.: Symbolic approach for measuring temporal "irreversibility", Phys. Rev. E, 62, 1912 1921, 2000.

Diks, D., van Houwelingen, J. C., Takens, F., and DeGoede, J.: Reversibility as a criterion for discriminating time series, Phys. Lett. A, 201, 221-228, 1995.

Farmer, J. and Sidorowich, J.: Predicting chaotic time series, Phys. Rev. Lett., 59(4), 845-848, 1987.

Goodrich, D. C., Lane, L. J., Shillito, R. M., and Miller, S. N.: Linearity of basin response as a function of scale in a semiarid watershed, Water Resour. Res., 33, 2951-2965, 1997.

Hegger, R., Kantz, H., and Schreiber, T.: Practical implementation of nonlinear time series methods: the TISEAN package, Chaos, 9(2), 413-435, 1999.

Kantz, H. and Schreiber, T.: Nonlinear Time Series Analysis, Cambridge University Press, Cambridge, England, 1997.

Kaplan, D. T.: Exceptional events as evidence for determinism, Physica D, 73, 38-48, 1994.

Kugiumtzis, D.: Statistically transformed autoregressive process and surrogate data test for nonlinearity, Phys. Rev. E, 66, 025 201-1-025 201-4, 2002.

Laio, F., Porporato, A., Ridolfi, L., and Rodriguez-Iturbe, I.: Mean first passage times of processes driven by white shot noise, Phys. Rev. E, 63(3), 036 105, 2001.

Lawrance, A. and Kottegoda, N.: Stochastic modeling of riverflow time series, Proc. Royal Stat. Soc. A, 140(1), 1-47, 1977.

Liu, C. C. and Brutsaert, W.: A nonlinear analysis of the relationship between rainfall and runoff for extreme floods, Water Resour. Res., 14, 75-83, 1978.

Livina, V. N., Ashkenazy, Y., Braun, P., Monetti, R., Bunde, A., and Havlin, S.: Nonlinear volatility of river flux fluctuations, Phys. Rev. E, 67, 042 101, 2003.

Murrone, F., Rossi, F., and Claps, P.: Conceptually-based shot noise modeling of streamflows at short time intervals, Stoch. Hydrol. Hydraul., 11, 483-510, 1997.

Pilgrim, D. H.: Travel times and nonlinearity of flood runoff from tracer measurements on a small watershed, Water Resour. Res., 12, 487-496, 1976.

Pilgrim, D. H. and Cordery, I.: Flood runoff, in: Handbook of Hydrology, edited by: Maidment, D. R., McGraw-Hill, New York, USA, 1993.

Porporato, A. and Ridolfi, L.: Nonlinear analysis of river flow time sequences, Water Resour. Res., 33, 1353-1367, 1997.

Porporato, A. and Ridolfi, L.: Detecting determinism and nonlinearity in river flow time series, Hydrol. Sci. J., 48, 763-780, 2003.

Rao, A. R. and Yu, G.-H.: Gaussianity and linearity tests of hydrological time series, Stochast. Hydrol. Hydraul., 4, 121-134, 1990.

Robinson, J. S., Sivapalan, M., and Snell, J. D.: On the relative roles of hillslope processes, channel routing, and network geomorphology in the hydrological response of natural catchements, Water Resour. Res., 31, 3089-3101, 1995.

Rogers, W. F.: Some characteristics and implications of drainage basin linearity and non-linearity, J. Hydrol., 55, 247-265, 1982.

Salas, J. D.: Analysis and modeling of hydrologic time series, in Handbook of Hydrology, edited by: Maidment, D. R., McGrawHill, New York, USA, 1993. 
Sauer, T., Yorke, J. A., and Casdagli, M.: Embedology, J. Statist. Phys., 65, 579-616, 1991.

Schreiber, T.: Interdisciplinary applications of nonlinear time series methods, Phys. Rep., 308, 1-64, 1999.

Schreiber, T. and Schmitz, A.: Discrimination power of measures for nonlinearity in a time series, Phys. Rev. E, 55, 5443-5447, 1997.

Schreiber, T. and Schmitz, A.: Surrogate time series, Physica D, 142, 346-382, 2000.

Sivakumar, B.: Chaos theory in hydrology: important issues and interpretations, J. Hydrol., 227, 1-20, 2000.

Sivakumar, B.: Chaos theory in geophysics: past, present and future, Chaos, Solitons and Fractals, 19, 441-462, 2004.

Takens, F.: Detecting stange attractors in turbulence, in: Lecture Notes in Mathematics, Vol. 898, Springer-Verlag, Berlin, Germany, 1981.
Theiler, J., Linsaly, P. S., and Rubin, D. M.: Exploring the continuum between deterministic and stochastic modeling, in: Time series prediction: forecasting the future and understanding the past, edited by: Weigend, A. and Gershenfeld, N., Addison-Wesley, Reading, MA, 1993.

Wang, C. T., Gupta, V. K., and Waymire, E.: A geomorphological synthesis of nonlinearity in surface runoff, Water Resour. Res., 17, 545-554, 1981.

Weiss, G.: Time-reversibility of nonlinear stochastic processes, J. Appl. Prob., 12, 831-836, 1975.

Weiss, G.: Shot noise models for the generation of synthetic stremflow data, Water Resour. Res., 13(1), 101-108, 1977. 